\title{
Cycle cellulaire, cancer, sénescence et p53
}

Il est bien justifié que la protéine p53 ait été désignée "molécule de l'année", par la revue Science. En réalité, cette protéine aurait déjà pu prétendre à une telle reconnaissance en 1991 et $1992\left(\mathrm{~m} / \mathrm{s} n^{\circ} 1\right.$, vol. 9, p. 79). On sait qu'elle est un régulateur de la prolifération cellulaire, bloquant le passage des cellules de la phase quiescente à la phase $\mathrm{S}$ de synthèse d'ADN. Dans certaines conditions, elle peut également induire l'apoptose cellulaire [1, 2] $\left(\mathrm{m} / \mathrm{s} n^{\circ} 9\right.$, vol. 8, p. 1002). La protéine p53 est induite en cas de dommages de l'ADN provoqués par des radiations ionisantes, des ultraviolets ou certains mutagènes [3] $\left(\mathrm{m} / \mathrm{s} n^{\circ} 1, \mathrm{vol}\right.$. 8 , p. 100). On suppose alors que le blocage de la synthèse d'ADN permet de procéder aux réparations nécessaires de cet $\mathrm{ADN}$ avant que d'en entreprendre la réplication. Le gène p53 est structuralement modifié dans environ $50 \%$ des cancers humains, constituant par conséquent la lésion moléculaire la plus fréquemment associée à ces maladies. I e rôle de p53 ne semble pas être d'intervenir, à l'état de base, dans le cycle cellulaire : l'invalidation par recombinaison homologue du gène $p 53$ ne nuit pas à un développement embryonnaire parfaitement normal chez des animaux homozygotes $\left(m / s \quad n^{\circ} 5\right.$, vol. 8, p. 492). En revanche, p53 constitue très certainement un système de sécurité, permettant, par exemple, la réparation des altérations de l'ADN ou provoquant l'apoptose des cellules ainsi altérées, système dont la défaillance provoque une importante susceptibilité au cancer [4]. La protéine p53 est un facteur de transcription dont la capacité de se fixer à ses cibles d'ADN est étroitement contrôlée par son état d'oligomérisation (des dimères ou, pour certains, des tétramères de p53 semblent être les formes se fixant à l'ADN), et par son aptitude à subir des transconformations de type allostérique. Les mutations faux-sens de la protéine p53 associées au cancer perturbent cette flexibilité, empêchant p53 d'adopter sa conformation ayant de l'affinité pour sa cible d'ADN $\left(m / s \quad n^{\circ} 6\right.$, vol. $8, p .612)$. Outre sa fixation spécifique sur une séquence particulière d'ADN, p53 peut également interagir avec TBP (TATA binding protein) inhibant ainsi la transcription d'une grande variété de promoteurs $\left(m / s n^{\circ} 2\right.$, vol. 9, p. 230). L'activité de p53 est modulée par interaction de diverses molécules, notamment le produit du gène $m d m 2$, amplifié dans certaines formes de cancer. Le complexe p53/mdm2 a perdu la capacité d'activer la transcription $\left(\mathrm{m} / \mathrm{s} \quad n^{\circ} 8 / 9\right.$, vol. 9, p. 998). A l'inverse, l'interaction de p53 avec certaines protéines fixant le calcium ou avec des anticorps reconnaissant spécifiquement sa région carboxyterminale, impliquée dans l'oligomérisation, stabilise les conformations capables de se lier spécifiquement à l'ADN (m/s $n^{\circ} 2$, vol. 9, p. 221).

A tout cet édifice considérable concernant le rôle et les propriétés de p53 manquait, jusqu'au mois de novembre 1993, un élément essentiel : quel(s) gène(s) cellulaire(s) est(sont) le(s) relais de ce facteur de transcription allostérique qu'est p53? C'est la convergence de deux programmes de recherche complètement indépendants qui a permis de commencer à combler cette lacune. Le laboratoire de P. Vogelstein avait précisément comme objectif de trouver des gènes contrôlés par p53. Pour ce faire, les chercheurs sont partis de lignées cellulaires exprimant, soit une version mutée de p53, dépourvue de pouvoir d'activation transcriptionnelle, soit un transgène de p53 intact placé sous le contrôle d'un promoteur inductible par les glucocorticoīdes. Après induction par cette hormone, un criblage différentiel des
ADNc issus de ces deux lignées a été réalisé, afin de détecter des clones correspondant à des messagers présents dans la lignée possédant le gène p53 intact et absents de l'autre lignée. Ainsi a été isolé le gène $W A F 1$. Ce gène est, en effet, stimulé dans sa transcription par p53 et son hyperexpression est capable de bloquer la prolifération cellulaire, plus particulièrement le passage de la phase Gl à la phase $\mathrm{S}$ du cycle cellulaire [5]

L'objectif de J.W. Harper et al., de Houston (TX) et Boston (MA, USA) était tout différent: il s'agissait d'identifier des régulateurs de protéine kinases dépendantes des cyclines (Cdk, cyclin-dependent kinase). La mieux connue de ces kinases est Cdc2 qui, associée à la cycline B, est indispensable à la mitose. Il existe, néanmoins bien d'autres types de Cdk et bien d'autres cyclines dont certaines sont spécifiques du passage de la phase $\mathrm{Gl}$ à la phase $\mathrm{S}$, et par conséquent du démarrage de la réplication de l'ADN. Tel est le cas de Cdk2, associée à l'une ou l'autre des cyclines caractéristiques de la phase Gl [6]. La stratégie de l'équipe dirigée par SJ Elledge fut de cloner des protéines capables d'interagir physiquement avec la kinase Cdk2. Pour ce faire, fut employée la méthode dite "des doubles hybrides" dans la levure Saccharomyces crevisiae. Le principe de cette approche, qui se révèle jour après jour d'une extraordinaire puissance, est le suivant: une souche de levure est transformée avec un gène de sélection placé sous le contrôle d'un élément de réponse au facteur transcriptionnel GAL4 et avec un vecteur d'expression commandant la synthèse d'une protéine hybride constituée de l'élément de fixation à l'ADN de la protéine GAL4 et de la protéine dont on cherche le partenaire. Dans les levures recombinantes ainsi obtenues, on transfère 


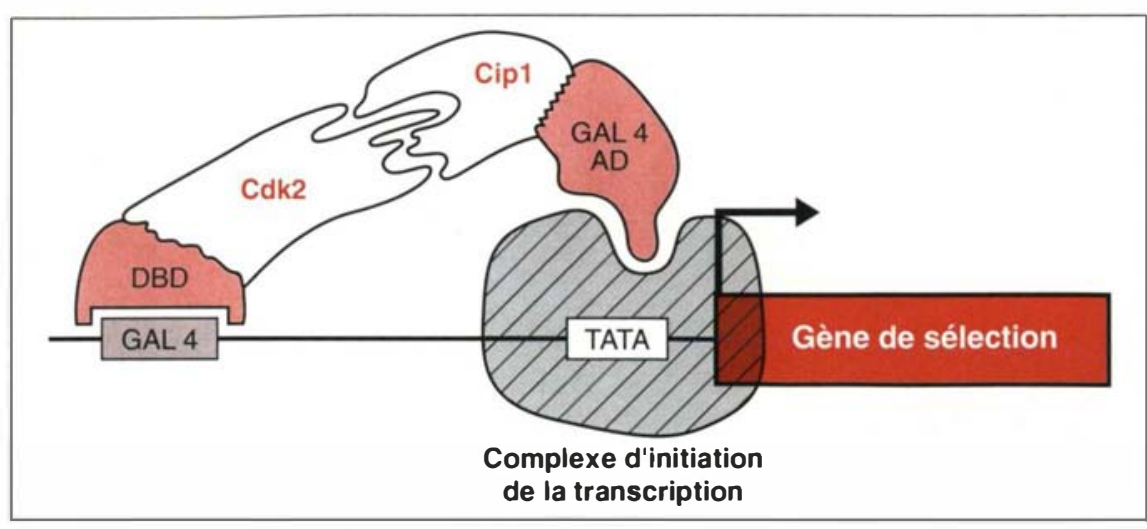

Figure 1. Clonage de Cip 1 par la technique des doubles hybrides chez la levure. Toutes les levures contiennent le gène de sélection sous le contrôle d'un élément de réponse à GAL-4 et synthétisent une protéine hybride composée du domaine de liaison à l'ADN de la protéine GAL4 IDBD, DNA binding domain) et de Cdk2. Des vecteurs contenant un $A D N c$ quelconque d'une banque d'ADNc lié au domaine d'activation (AD) de GAL 4 sont transfectés dans ces levures. Les levures synthétisant Cip1, un partenaire d'interaction avec Cdk2, lié au domaine d'activation transcriptionnelle de GAL 4, expriment le gène de sélection grâce auquel elles peuvent être sélectionnées.

secondairement des plasmides dans lesquels ont été clonés des $\mathrm{ADN}$ complémentaires juxtaposés à la séquence codant pour le domaine activateur du facteur de transcription GAL4. Les levures synthétisant le partenaire protéique de la molécule étudiée, couplé à un domaine d'activation transcriptionnel, transcrivent activement le gène de sélection et peuvent ainsi être reconnues (figure 1). J.W. Harper et al. isolèrent ainsi la protéine Cip 1 (Cdk2 inhibitory protein 1) capable de former un complexe avec Cdk2 et d'en inhiber l'activité de protéine-kinase, bloquant ainsi la transition Gl $\rightarrow$ S. Cet arrêt du cycle cellulaire est capable de s'opposer au pouvoir activateur de la prolifération de l'oncogène $T$ du virus SV 40 [6]. La même protéine a été étudiée sous d'autres noms par d'autres équipes américaines $[7,8]$, et l'ADNc correspondant a été cloné par l'une d'entre elles [7]. Cette dernière équipe, dirigée par D. Beach (Cold Spring Harbor, NY, USA), a étendu à d'autres kinases de la famille Cdk la recherche des inhibiteurs protéiques: pl $6^{\text {INK4 }}$ inhibe ainsi le complexe Cdk4/cycline D dont l'un des rôles pourrait être de phosphoryler les $\mathrm{m} / \mathrm{s} n^{\circ} 2$ vol. 10 , férico 94 ment cellulaire [10]. Dénommé alors sdi 1 (senescent cell-derived inhibitor 1), ce gène est actif dans les cellules sénescentes et peut bloquer la synthèse d'ADN. Si ces résultats, sous presse dans la revue Experimental Cell Research, se confirmaient, p53 apparaîtrait alors doté d'un autre rôle de contrôle que ceux évoqués ci-dessus : celui de la longévité cellulaire

A.K.

\section{RÉFÉRENCES}

1. Kahn A, Briand P. Apoptose, une mort programmée ou une prolifération avortée? médecine/sciences 1993: 9:663-5.

2. Lowe SW, Ruley HE, Jacks T, Housman DE. p53-dependent apoptosis modulates the cytotoxicity of anticancer agents. Cell $1993 ; 74: 957-67$.

3. Lu X, Lane DP. Differential induction of transcriptionally active $\mathrm{p} 53$ following UV or ionizing radiation: defects in chromosome instability syndromes? Cell 1993; 75 : 765-78.

4. Harvey M, McArthur MJ, Montgomery CA, Butel JS, Bradley A, Donehower LA Spontaneous and carcinogen-induced tumorigenesis in p53-deficient mice. Nature Genel 1993, 5 : 225-9.

5. El-Deiry WS, Tokino T, Velculescu VE Levy DB, Parsons R, Trent JM, Lin D, Mercer WE, Kinzler KW, Vogelstein B. WAFI, a potential mediator of p53 tumor suppression. Cell 1993; 75: 817-25.

6. Harper JW, Adami GR, Wei N, Keyormarsi K, Elledge SJ. The p2 I Cdkinteracting protein Cipl is a potent inhibitor of G1 cyclin-dependent kinases. Cell $1993 ; 75: 805-16$.

7. Xiong Y, Hannon (J), Zhang $\mathrm{H}$, Casso D, Kobayaski R, Beach D. p21 is a universal inhibitor of cyclin kinases. Nature 1993, 366: $701-4$.

8. Gu Y, Turck CW, Morgan DO. Inhibition of CDK2 activity in vivo by an associated $20 \mathrm{~K}$ regulatory subunit. Nature $1993 ; 366: 707-10$.

9. Serrano M, Hannon GJ, Beach D. A new regulatory motif in cell cycle control causing specific inhibition of cyclin D/CDK 4. Nature 1993; $366: 704-7$.

10. Marx J, How p53 suppresses cell growth. Science 1993; 262: 1644-5. rétablir l'intégrité de l'ADN, et d'éviter ainsi l'apparition de mutations dont certaines pourraient aboutir à l'activation d'oncogènes.

Le gène $p 53$ et sa cible, WAF1/CIP1 pourraient enfin être impliqués dans l'une des manifestations essentielles de la sénescence $e x$ vivo, la perte de la capacité mitotique. En effet, il semble que WAF1/CIP1 ait été déjà cloné, il y a plus de deux ans, par des biologistes étudiant le vieillisse-

\section{Axel Kahn \\ Directeur de l'unité de recherches en généti que et pathologie moléculaires, Inserm U 129. ICGM, 24, rue du Faubourg Saint-Jacques, 75014 Paris, France.}

\section{TIRÉS A PART}

